\title{
The Planck Constant and the Origin of Mass due to a Higher Order Casimir Effect
}

\author{
C. Baumgärtel and M. Tajmar* \\ Institute of Aerospace Engineering, Technische Universität Dresden, Germany
}

(Dated: May 31, 2018)

\begin{abstract}
The Planck constant is one of the most important constants in nature, as it describes the world governed by quantum mechanics. However, it cannot be derived from other natural constants. We present a model from which it is possible to derive this constant without any free parameters. This is done utilizing the force between two oscillating electric dipoles described by an extension of Weber electrodynamics, based on a gravitational model by Assis. This leads not only to gravitational forces between the particles but also to a newly found Casimir-type attraction. We can use these forces to calculate the maximum point mass of this model which is equal to the Planck mass and derive the quantum of action. The result hints to a connection of quantum effects like the Casimir force and the Planck constant with gravitational ones and the origin of mass itself.
\end{abstract}

Keywords: Planck Constant, Casimir Force, Quantum Gravity, Unified Field Theory, Weber Electrodynamics, Origin of Mass

\section{INTRODUCTION}

The quantum of action $h$ is one of the fundamental constants of nature. Originally assumed to calculate the black body radiation, its value has been determined experimentally to a high degree of certainty. It is most important to describe the phenomena of quantum theory and marks a minimum increment of energy of an electrically charged oscillating particle in the atomic scale. However, it cannot be derived or calculated from other constants.

In an earlier work, Tajmar attempted an explanation of Planck's constant [1] using a gravitational model of dipoledipole interaction by Assis [2, 3] based on Weber electrodynamics [4]. A major drawback of his result was, that to arrive at the correct value of $h$, there was still an unknown constant necessary. Nonetheless, he managed to arrive at the correct order of magnitude and showed a possible connection between Weber's theory and quantum theory. Based on this idea, the calculations of Assis [2, 3] were redone and we will show a method to derive Planck's constant without any free parameters. This was achieved through a newly found connection to the Casimir force in Weber's force, which also hints to quantum effects being related to Weber electrodynamics.

Historically, Weber's theory pre-dates Maxwell's publications [5] by 15 years and belongs to the action-at-a-distance theories that need no medium of interaction, contrary to the fieldapproach. The field concept replaced the aether and is a basic part of today's standard model in physics, whilst action-at-adistance theories are often dismissed ad-hoc. But from today's point of view, Weber's equation can withstand all criticism that arose historically and led to its rejection, as shown in [68]. Recently, there is a new spark of interest on this topic, with Assis being one of the first to reintroduce the matter. Researchers have made different attempts not only to modify Weber's law and derive Maxwell's field equations from them [8-10], but also conducted experiments to check back on the validity of the theory [11-14]. Especially the experiments of Smith et al. [12] showed some interesting measurements where the behaviour of an electron beam could

*martin.tajmar@tu-dresden.de; corresponding author be predicted more accurately with Weber-type formulae than Maxwell-Lorentz ones.

This interest leads us to study further on this subject and investigate the bonds that connect Weber's law and nature's phenomena, as this may be a possibility to find a long sought after unification of theories. Therefore, we will present our findings that combine electromagnetism, gravitational-like forces, quantum effects and even the origin of mass itself all through the derivation of the Planck constant from a single model of oscillating dipoles.

In the Higgs-mechanism [15, 16], all massive particles are explained to have a mass that originates from their interaction with the Higgs-field. This includes the $\mathrm{W}$ - and Z-Bosons, but what remains unresolved is the origin of the Higgs-Boson's mass itself. With the model presented in this paper, we show how the interaction of massless particles creates massproperties of the particles themselves.

\section{EXTENDED WEBER ELECTRODYNAMICS}

To show how mass and Planck's constant may be obtained through this concept, we give a short summary of the model originally used by Assis [2,3] and point out the differences in the new approach. The basic concept is, that a massive particle consists of an oscillating dipole. With the Weber force, there exists a non-zero attractive force between two of these dipoles which is along the line joining them and falls with $1 / r^{2}$, like gravity does.

\section{A. Gravitational Model by Assis}

Firstly, let us revisit the calculations done by Assis [2, 3] and explain how the model works. We need to look at the Weber potential energy between two charges $q_{1}$ and $q_{2}$ like in Figure 1 , depending only on their relative distance and velocity, and given as

$$
U=\frac{q_{1} q_{2}}{4 \pi \varepsilon_{0}} \frac{1}{r_{12}}\left(1-\frac{\dot{r}_{12}^{2}}{2 c^{2}}\right)
$$




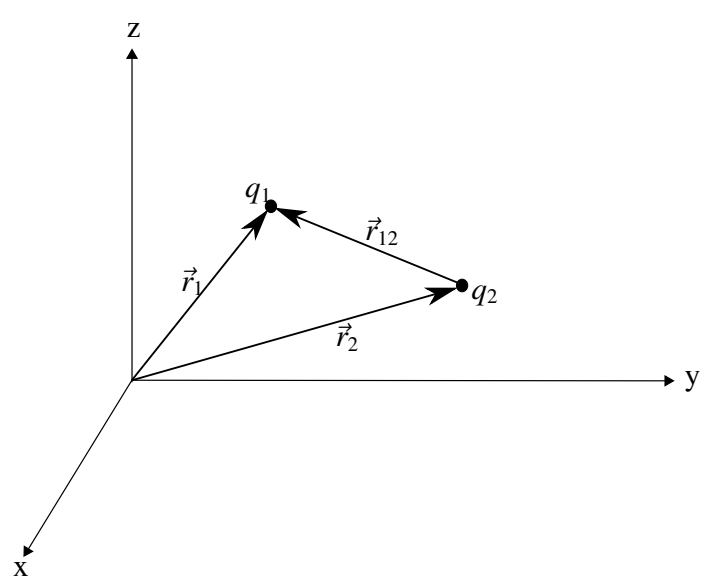

FIG. 1. Positions of two charges $q_{1}$ and $q_{2}$ in a frame of reference

where

$$
\begin{aligned}
r_{12} & =\left|\vec{r}_{12}\right|=\left|\vec{r}_{1}-\vec{r}_{2}\right| \\
& =\sqrt{\left(x_{1}(t)-x_{2}(t)\right)^{2}+\left(y_{1}(t)-y_{2}(t)\right)^{2}+\left(z_{1}(t)-z_{2}(t)\right)^{2}}
\end{aligned}
$$

$$
\dot{r}_{12}=\hat{r}_{12} \cdot \vec{v}_{12}
$$

with

$$
\begin{gathered}
\hat{r}=\frac{\vec{r}_{12}}{r_{12}} \\
\frac{d \vec{r}_{12}}{d t}=\vec{v}_{12}
\end{gathered}
$$

as well as $\varepsilon_{0}$ being the vacuum permittivity and $c$ the speed of light.

Equation (1) is then extended by Assis [2, 3] as a seriesexpansion to a general form, such that

$$
U=\frac{q_{1} q_{2}}{4 \pi \varepsilon_{0}} \frac{1}{r_{12}}\left[1-\frac{\alpha}{c^{2}} \dot{r}_{12}^{2}-\frac{\beta}{c^{4}} \dot{r}_{12}^{4}-\frac{\gamma}{c^{6}} \dot{r}_{12}^{6}-\ldots\right]
$$

and derived through the concept of virtual work

$$
\vec{F}_{21}=-\hat{r}_{12} \frac{d U}{d r_{12}} \quad \text { or } \quad \vec{v}_{12} \vec{F}_{21}=-\frac{d U}{d t}
$$

to get the extended Weber force

$$
\begin{aligned}
\vec{F}_{21}=\frac{q_{1} q_{2}}{4 \pi \varepsilon_{0}} \frac{\hat{r}_{12}}{r_{12}^{2}}( & 1-\frac{\alpha}{c^{2}}\left(\dot{r}_{12}^{2}-2 r_{12} \ddot{r}_{12}\right)-\frac{\beta}{c^{4}}\left(\dot{r}_{12}^{4}-4 \dot{r}_{12}^{2} r_{12} \ddot{r}_{12}\right) \\
& \left.-\frac{\gamma}{c^{6}}\left(\dot{r}_{12}^{6}-6 \dot{r}_{12}^{4} r_{12} \ddot{r}_{12}\right)-\ldots\right)
\end{aligned}
$$

where

$$
\dot{r}_{12}=\hat{r}_{12} \cdot \vec{v}_{12}
$$

$$
\ddot{r}_{12}=\frac{\left[\vec{v}_{12} \cdot \vec{v}_{12}-\left(\hat{r}_{12} \cdot \vec{v}_{12}\right)^{2}+\vec{r}_{12} \cdot \vec{a}_{12}\right]}{r_{12}}
$$

With this equation and its newly introduced constants $\alpha, \beta, \gamma$, we can derive all electrostatic and electrodynamic phenomena for $\alpha=0.5$ and $\beta=\gamma=0$. For instance, the Coulomb force is then easily obtained if the charges are not moving, meaning $\dot{r}_{12}=\ddot{r}_{12}=0$.

This force is now used to describe the interaction of two oscillating dipoles, where the positive charge $q_{+}$is in the centre of the dipole and the negative charge $q_{-}$is moving along one dimension with $A_{-} \sin (\omega t+\theta)$. The basic idea here is, that there is a non-zero resultant force between the dipoles, since the charges of a dipole are interacting with every charge of the other dipole. The oscillation is shown in Fig. 2 for both dipoles oscillating in the $\mathrm{x}$ direction. In this case we get the

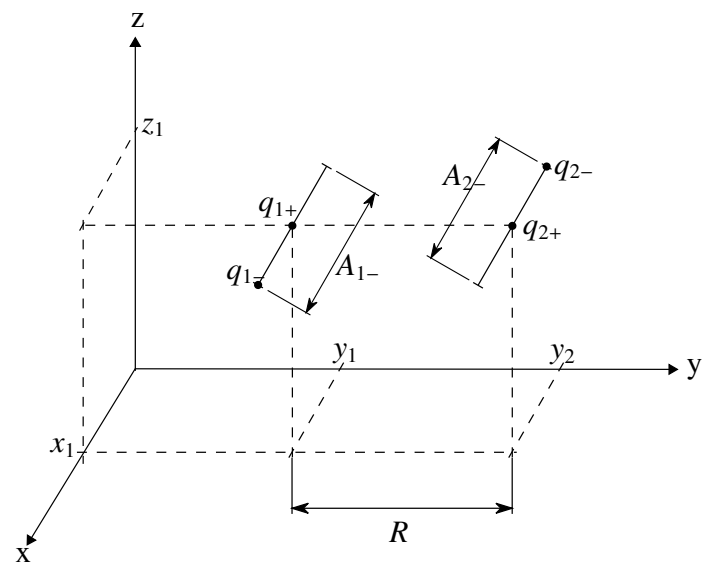

FIG. 2. Dipole 1 and dipole 2 both oscillate along the $\mathrm{x}$-axis

position of each of the charges as follows:

$$
\begin{gathered}
\vec{r}_{1+}=x_{1} \hat{x}+y_{1} \hat{y}+z_{1} \hat{z}, \quad \vec{r}_{2+}=x_{1} \hat{x}+y_{2} \hat{y}+z_{1} \hat{z} \\
\vec{r}_{1-x x}=\left[x_{1}+A_{1-} \sin \left(\omega_{1} t+\theta_{1}\right)\right] \hat{x}+y_{1} \hat{y}+z_{1} \hat{z} \\
\vec{r}_{2-x x}=\left[x_{1}+A_{2-} \sin \left(\omega_{2} t+\theta_{2}\right)\right] \hat{x}+y_{2} \hat{y}+z_{1} \hat{z}
\end{gathered}
$$

Note that in this case, we assume that the dipoles are only separated along the $y$-axis. The expressions $\hat{x}, \hat{y}$ and $\hat{z}$ are the unit vectors of the corresponding axes. To get a homogenous distribution, Assis allows each of the dipoles to oscillate in either $\mathrm{x}-, \mathrm{y}-$ or $\mathrm{z}$-direction independently from the other, so that we get nine possible cases how the dipoles can be oriented. Fig. 3 shows another of these possibilities, where the first dipole oscillates in the y-direction and the second dipole in $\mathrm{z}$-direction. The respective positions of the negative charges are then described by

$$
\begin{aligned}
& \vec{r}_{1-y z}=x_{1} \hat{x}+\left[y_{1}+A_{1-} \sin \left(\omega_{1} t+\theta_{1}\right)\right] \hat{y}+z_{1} \hat{z} \\
& \vec{r}_{2-y z}=x_{1} \hat{x}+y_{2} \hat{y}+\left[z_{1}+A_{2-} \sin \left(\omega_{2} t+\theta_{2}\right)\right] \hat{z}
\end{aligned}
$$

while Eq. (11) holds true, since the positive charges remain fixed with respect to the oscillation. The dipoles in general can 


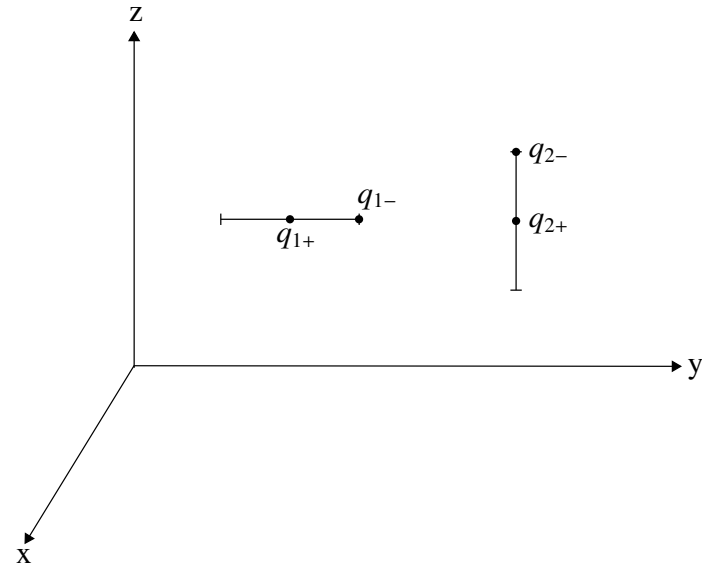

FIG. 3. Oscillation of the first dipole along the y-axis and the second along the $\mathrm{z}$-axis

however move with respect to each other, since the positions $x_{1,2}, y_{1,2}, z_{1,2}$ are a function of time and we already assumed them to be only different along the y-axis. We will simplify this behaviour even more in the next step.

The nine cases are essential to calculate the average resulting force between the dipoles, but before further explanation of how this is done, we want to give a quick overview of the assumptions taken by Assis, which are listed below.

- $x_{1}=x_{2}, \quad z_{1}=z_{2}, \quad y_{1}-y_{2}=R$

- $q_{1-}=-q_{1+}, \quad q_{2-}=-q_{2+}$

- $A_{1+}=A_{2+}=0, \quad A_{1-} \neq 0, \quad A_{2-} \neq 0$

- $A_{1}^{2} / R^{2} \ll 1, \quad A_{2}^{2} / R^{2} \ll 1$

- $R^{2} \gg c^{2} / \omega_{1}^{2}, \quad R^{2} \gg c^{2} / \omega_{2}^{2}$

- $R^{2} \omega_{1}^{2} \gg V^{2} \gg A_{1}^{2} \omega_{1}^{2}$

- $R^{2} \omega_{2}^{2} \gg V^{2} \gg A_{2}^{2} \omega_{2}^{2}$

- $R^{2} \omega_{1}^{2} \gg R A \gg A_{1}^{2} \omega_{1}^{2}$

- $R^{2} \omega_{2}^{2} \gg R A \gg A_{2}^{2} \omega_{2}^{2}$

- $\omega_{1}=\eta \omega_{2}, \quad \eta=1 ; 2 ; 3 ; \ldots$

- $T_{2}=2 \pi / \omega_{2}$

- $\vec{R}, \vec{V}, \vec{A}$ are constant between $t=0 \ldots T_{2}$

Here we have additional variables, that define the relation of the dipole centres, with the relative position $\vec{R}=\left(x_{1}-x_{2}\right) \hat{x}+$ $\left(y_{1}-y_{2}\right) \hat{y}+\left(z_{1}-z_{2}\right) \hat{z}=R_{x} \hat{x}+R_{y} \hat{y}+R_{z} \hat{z}$, velocity $\vec{V}=d \vec{R} / d t$ and acceleration $\vec{A}=\vec{V} / d t$. And their absolute values $R=|\vec{R}|$, $V=|\vec{V}|, A=|\vec{A}|$, where in the assumed case of the dipoles being only separated in the y-direction $R_{y}=R$. Furthermore, there is a highest period $T_{2}$ and the relative movement of the dipoles in this time interval is constant, if not negligible in this period. It is also assumed, that the oscillation-frequency $\omega_{1}$ is an integral multiple of $\omega_{2}$. This is done to perform an average over time in the calculation and we will explain now, how the resulting force is computed.

Equation (8) is utilized up to the sixth order term and $\beta$ and $\gamma$ are not zero in this case. For each of the nine cases we can calculate a sum of four forces, where each of the forces exists between one pair of charges made up of one charge of either dipole, since a single charge in a dipole is interacting with every charge of the other dipole.

$$
\vec{F}=\vec{F}_{2+, 1+}+\vec{F}_{2+, 1-}+\vec{F}_{2-, 1+}+\vec{F}_{2-, 1-}
$$

Therefore the corresponding positions $\vec{r}_{1+}, \vec{r}_{2+}, \vec{r}_{1-}, \vec{r}_{1-}$ of one orientation at a time are inserted into Eq. (8). Because only the average force is of interest, three averages are performed in the next step. These are two averages over the phases $\theta_{1}$ and $\theta_{2}$ with values between 0 and $2 \pi$ for both, and one over time from 0 to $T_{2}$, see Eq. (17).

$$
\bar{F}=\frac{1}{T_{2}} \int_{0}^{T_{2}} d t \frac{1}{2 \pi} \int_{0}^{2 \pi} d \theta_{1} \frac{1}{2 \pi} \int_{0}^{2 \pi} d \theta_{2} \vec{F}
$$

The resulting forces of each case are then added and divided by nine as a fourth average and lead to the final mean force between two oscillating dipoles. In [2, 3], Assis gives this result, after generalising it, as

$$
\vec{F}=-\frac{7 \beta}{18} \frac{q_{1+} q_{2+}}{4 \pi \varepsilon_{0}} \frac{\vec{R}}{R^{3}} \frac{A_{1-}^{2} \omega_{1}^{2} A_{2-}^{2} \omega_{2}^{2}}{2 c^{4}}\left(1+\frac{\gamma}{\beta} \frac{45 \dot{R}^{2}-18 R \ddot{R}}{7 c^{2}}\right)
$$

where $\dot{R}=d R / d t=\vec{R} \cdot \vec{V} / R$ and $\ddot{R}=d^{2} R / d t^{2}=d \dot{R} / d t=$ $\left(\vec{V} \cdot \vec{V}-(\vec{R} \cdot \vec{V} / R)^{2}+\vec{R} \cdot \vec{A}\right) / R$. This equation is used in [2, $3]$ to identify a gravitational effect and this is again used in [1] to obtain a constant in the order of magnitude of Planck's constant.

The above presented calculations were done by Assis with pen and paper and are very long. This is why we wanted to check the results using a computer, so the calculations were redone in MAXIMA and showed a slightly different result. Starting from the nine cases, the steps of the procedure were followed with a few extra-assumptions. Here, the dipole centres are not moving relative to one another, meaning

$$
\text { - } \vec{V}=0, \quad \vec{A}=0
$$

and according to $A^{2} / R^{2} \ll 1$ all terms higher than $A^{4}$ are dismissed. This was necessary to obtain a result of reasonable length and is justifiable due to the fact, that the attractive effect that we want to observe is also present if the dipoles are not in motion. Thus, our new result is Eq. (19).

$$
\begin{aligned}
\bar{F}=-\frac{q_{1+} q_{2+}}{576 \pi \varepsilon_{0}} \frac{R_{y} \hat{y}}{R^{5}}[ & \frac{\alpha}{c^{2}} 60 A_{1-}^{2} A_{2-}^{2}\left(\omega_{1}^{2}+\omega_{2}^{2}\right) \\
& +\frac{\beta}{c^{4}} 152 A_{1-}^{2} A_{2-}^{2} \omega_{1}^{2} \omega_{2}^{2} R^{2} \\
& \left.+\frac{\gamma}{c^{6}} 369 A_{1-}^{2} A_{2-}^{2} \omega_{1}^{2} \omega_{2}^{2}\left(A_{1-}^{2} \omega_{1}^{2}+A_{2-}^{2} \omega_{2}^{2}\right) R^{2}\right]
\end{aligned}
$$

We can see, that the pre-factors of the terms are different and that there is a new term that was not present before. 
Assis identified in $[2,3]$ that the $\beta$-term corresponds to a gravitational attraction and the $\gamma$-term to inertial effects. We will further analyse this in section III. It should also be mentioned, that we were able to get the same pre-factor for the $\beta$-term as in Eq. (18) when the second derivative of $r_{12}$ was calculated without time dependence. This confirms Assis' analytical model for $\beta$.

\section{B. 3D model - Orientation in three Dimensions}

In the new approach, the model is slightly expanded, though keeping the original one-dimensional oscillation of the dipoles. We now want to study the average force, if the orientation is not limited to the nine cases but can happen in every possible direction in three-dimensional space, as long as the oscillation is one-dimensional (see Fig. 4). A normal vector $\vec{n}$

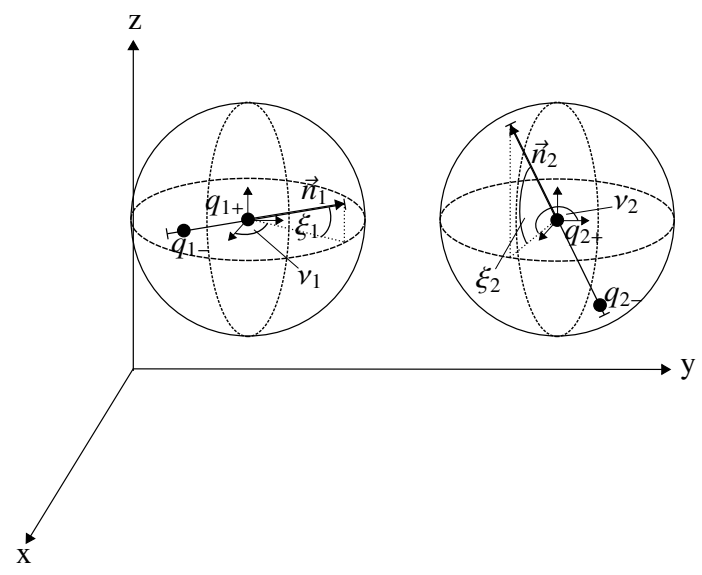

FIG. 4. Orientation of the dipoles in every possible direction

is defined to point from the centre of the dipole to a position on the surface of a sphere, thus describing the direction of the negative charge's oscillation.

$$
\vec{n}_{1,2}=\left(\begin{array}{c}
\cos \left(v_{1,2}\right) \cos \left(\xi_{1,2}\right) \\
\sin \left(v_{1,2}\right) \cos \left(\xi_{1,2}\right) \\
\sin \left(\xi_{1,2}\right)
\end{array}\right)
$$

Here the variable $v$ is a longitudinal angle having any value between 0 and $2 \pi$, while the latitudinal angle $\xi$ gets replaced by

$$
\xi_{1,2}=\arcsin \left(b_{1,2}\right) \quad \text { mit } \quad b_{1,2} \in[-1 \ldots 1]
$$

because this ensures that every point in three-dimensional space is included with an even distribution. As an example, the negative charge of dipole one is then described as

$$
\vec{r}_{1-}=A_{1-} \sin \left(\omega_{1} t+\theta_{1}\right) \cdot\left(\begin{array}{c}
\cos \left(v_{1}\right) \cos \left(\xi_{1}\right) \\
\sin \left(v_{1}\right) \cos \left(\xi_{1}\right) \\
\sin \left(\xi_{1}\right)
\end{array}\right)+\vec{r}_{1+}
$$

To speed up the calculation and save computing power, two more assumptions were implemented.
- We only included terms up to the order $A^{2}$

- The $\gamma$-term is much smaller than the $\alpha$ - and $\beta$-terms and can be neglected

We can then calculate the sum of the four forces by inserting Eq. (22) and an analogue expression for the second dipole along with (11) into (8). The average will now be performed over the phases and also over the angles $v$ and $\xi$.

$$
\begin{aligned}
\bar{F}= & \frac{1}{2} \int_{-1}^{1} d \arcsin \left(b_{2}\right) \frac{1}{2 \pi} \int_{0}^{2 \pi} d v_{2} \frac{1}{2} \int_{-1}^{1} d \arcsin \left(b_{1}\right) . \\
& \cdot \frac{1}{2 \pi} \int_{0}^{2 \pi} d v_{1} \frac{1}{2 \pi} \int_{0}^{2 \pi} d \theta_{2} \frac{1}{2 \pi} \int_{0}^{2 \pi} d \theta_{1} \vec{F}
\end{aligned}
$$

This yields as the resulting force for the dipoles being allowed to oscillate one-dimensionally in every possible direction in three-dimensional space

$$
\begin{array}{r}
\bar{F}=-\frac{q_{1+} q_{2+} \hat{y}}{144 \pi \varepsilon_{0} R^{4}}\left(\frac{\alpha}{c^{2}} 15 A_{1-}^{2} A_{2-}^{2}\left(\omega_{1}^{2}+\omega_{2}^{2}\right)\right. \\
\left.+\frac{\beta}{c^{4}} 38 R^{2} A_{1-}^{2} A_{2-}^{2} \omega_{1}^{2} \omega_{2}^{2}\right)
\end{array}
$$

\section{DERIVING PLANCK'S CONSTANT THROUGH THE CASIMIR FORCE IN THE NEW MODEL}

If we look at the equations (18), (19) and (24) and compare them, we see that the new results differ from the one Assis achieved. Not only are there different pre-factors for the $\beta$ and $\gamma$-terms, the structure of the $\gamma$-term is different too. One reason is of course that we disregarded the movement of the dipole centres, but other than that it now depends on fourth order terms of the Amplitude, which was not the case with Assis' outcome.

Moreover, there is a new term correlating with $\alpha$ that was not present before. We can clearly see that the $\alpha$ - and $\beta$-terms are the exact same in Eq. (19) and (24), with 5/48 as the $\alpha$ pre-factor and $19 / 72$ for $\beta$ in both, which contributes to the plausibility of the new model. Furthermore, the $\beta$-term can be identified as a gravity-like force, as already done by Assis $[2,3]$, since only the pre-factor deviates but the structure is the same. Keeping in mind that the $\gamma$-term can be interpreted as inertial effects [2], what does the new $\alpha$-term correspond to? Since it falls with $1 / R^{4}$, it looks similar to the Casimir force [17] which originates from Van-der-Waals dipole-dipole interaction [18]. There were earlier approaches from Puthoff and Haisch to model gravity and inertia as zero-point energy fluctuations based on the Casimir effect [19-22], but they were heavily discussed afterwards [23-25].

However, it seems in agreement with Casimir's original assumptions to find his force in this model of dipole-dipole interactions. To summarize the interpretation of the results up to here, the following effects arise naturally in the model:

$1^{\text {st }}$ order: The Casimir force ( $\alpha$-term, falls with $\left.1 / r^{4}\right)$;

$2^{\text {nd }}$ order: Gravitational attraction $\left(\beta\right.$-term, falls with $\left.1 / r^{2}\right)$ and 
$3^{\text {rd }}$ order: Inertial effects ( $\gamma$-term, falls with $1 / r^{2}$ )

all in a single approach.

The first two orders of magnitude effects lead us to a renewed way to derive Planck's constant from the results. Following Tajmars model [1], we interpret the dipoles as vibrating strings that have elementary charges attached to either end. These "charged photons" would then move at the speed of light and limit the frequency of oscillation. It also appears in this model, that mass would need at least one vibrating dipole to be created, so a single charge on a string is massless. Such particles are discussed in $[26,27]$ and are shown to not radiate. We can then describe the string frequency as

$$
\omega=\frac{n \pi c}{A}
$$

where $n$ is the mode of the string vibration. To best represent our model, we take $n=2$ for one vibrating and one stationary end. We then identify the $\beta$-term to possess gravitational properties and one can write

$$
\frac{G m^{2}}{R^{2}}=\frac{19 e^{2} A^{4} \omega^{4} \beta}{72 \pi \varepsilon_{0} R^{2} c^{4}}
$$

This creates an expression for mass depending on the electrical properties of the strings as:

$$
m^{2}=\frac{19 n^{4} e^{2} \beta \pi^{3}}{72 G \varepsilon_{0}}
$$

Analogous to [1], this mass denotes the maximum possible point mass and can therefore be equalled to the Planck mass.

$$
m_{p}^{2}=\frac{h c}{2 \pi G}=\frac{19 n^{4} e^{2} \beta \pi^{3}}{72 G \varepsilon_{0}}
$$

Thus, we can model the Planck constant as

$$
h_{\text {model }}=\frac{19 n^{4} e^{2} \beta \pi^{4}}{36 c \varepsilon_{0}}
$$

Now we introduce the newly found Casimir force from the $\alpha$-term.

$$
F_{C_{\text {model }}}=\frac{5 e^{2} A^{4} 2 \omega^{2} \alpha}{48 \pi \varepsilon_{0} R^{4} c^{2}}
$$

The corresponding area of this force's action is given by the amplitude of the oscillation,

$$
A E=A^{2} \pi
$$

so we can formulate the Casimir pressure with $\alpha$ and $\beta$

$$
p_{C_{\text {model }}}=\frac{F_{C_{\text {model }}}}{A E} \frac{\varepsilon_{0}}{\varepsilon_{0}}=\frac{15 \alpha h c}{38 n^{2} \pi^{4} \beta R^{4}}
$$

This allows us to get a ratio of Eq. (32) with the known Casimir pressure (33).

$$
p_{\text {Casimir }}=\frac{\pi h c}{480 R^{4}}
$$

$$
\frac{p_{\text {Casimir }}}{p_{C_{\text {model }}}}=\frac{19 n^{2} \beta \pi^{5}}{3600 \alpha}
$$

which leads to an expression of $\beta$ as a function of $\alpha$.

$$
\beta_{\text {model }}=\frac{3600 \alpha}{19 n^{2} \pi^{5}}
$$

So far, we have only known the value for $\alpha=0.5$ from electromagnetism, but we can calculate $\beta$ for the first time to be

$$
\beta_{\text {model }} \approx 0.07739
$$

If we further insert eq. (35) in eq. (29) we can express Planck's constant as

$$
h_{\text {model }}=\frac{50 n^{2} e^{2}}{\pi \varepsilon_{0} c}
$$

With the known value of $h \approx 6.626 \times 10^{-34} \mathrm{~J} \mathrm{~s}$, we get a ratio of the modelled constant to Planck's constant of

$$
\frac{h_{\text {model }}}{h}=0.9291
$$

We can obtain approximately $93 \%$ of the quantum of action through this model without any free parameters! Despite a slight margin of $7 \%$ this is still a remarkable result, for there are still possible improvements if the assumptions are altered or loosened. There is also an interesting, relatively new approach by J. M. Montes to enforce finite velocities upon Weber dynamics [28], which could contribute to this model. Clearly these findings show a connection between Weber electrodynamics and quantum effects like the Casimir force and Planck's constant.

\section{CONCLUSION}

We showed that it is possible to derive the quantum of action up to $93 \%$ with a model of one-dimensionally oscillating dipoles utilizing extended Weber dynamics, and what's more it was achieved only through known values of parameters. Interestingly enough, this was possible by identifying the Casimir force in the model and using a higher order of this effect to calculate Planck's constant. Furthermore, this connects to the origin of mass as the higher order of the Casimir force shows a mass-like behaviour depending on the electrical properties of the interacting particles. These properties also lead to a gravitational-like attracting force between the particles. That means that there is a really interesting connection between electrodynamics, gravitation, quantum-theory and the origin of mass, all linked by the Planck constant.

\section{ACKNOWLEDGMENTS}

One of the authors (C. Baumgärtel) would like to thank G. Strey and S. Klioner for useful mathematical advice. 
[1] M. Tajmar, Journal of Advanced Physics 4, 219 (2015).

[2] A. K. T. Assis, Canadian Journal of Physics 70, 330 (1992).

[3] A. K. T. Assis, "Gravitation as a fourth order electromagnetic effect," in Advanced Electromagnetism: Foundations, Theory and Applications, edited by T. W. Barret and D. M. Gales (World Scientific Publishers, Singapore, 1995) pp. 314-331.

[4] W. E. Weber, Wilhelm Weber's Werke, edited by H. Weber, Vol. 3 (First part) (Julius Springer, Berlin, 1893).

[5] J. C. Maxwell, The London, Edinburgh, and Dublin Philosophical Magazine and Journal of Science 21, 161 (1861).

[6] A. K. T. Assis, "Arguments in favour of action at a distance," in Instantaneous Action at a Distance in Modern Physics- "Pro" and "Contra", edited by A. E. Chubykalo, V. Pope, and R. Smimov-Rueda (Nova Science Publishers, Commack, 1999) pp. $45-56$.

[7] A. K. T. Assis and H. T. Silva, Pramana 55, 393 (2000).

[8] A. K. T. Assis, "Weber's electrodynamics," (Springer, Dordrecht, 1994) pp. 47-77.

[9] E. Kinzer and J. Fukai, Foundations of Physics Letters 9, 457 (1996).

[10] J. P. Wesley, Foundations of Physics Letters 3, 443 (1990).

[11] V. F. Mikhailov, Annales de la Fondation Louis de Broglie 26, 633 (2001).

[12] R. T. Smith, F. P. Jjunju, and S. Maher, Progress In Electromagnetics Research 151, 83 (2015).
[13] I. Lőrincz and M. Tajmar, Canadian Journal of Physics 95, 1023 (2017).

[14] M. Weikert and M. Tajmar, "Investigation of the Influence of a Field-free Electrostatic Potential on the Electron Mass with Barkhausen-Kurz-Oscillation,” (2017), submitted to Annales de la Fondation Louis de Broglie.

[15] P. W. Higgs, Physical Review Letters 13, 508 (1964).

[16] P. W. Higgs, Physical Review 145, 1156 (1966).

[17] H. B. G. Casimir, Proc. Kon. Ned. Akad. Wertensch. 51, 793 (1948).

[18] H. Casimir and D. Polder, Physical Review 73, 360 (1948).

[19] H. E. Puthoff, Physical Review A 39, 2333 (1989).

[20] H. Puthoff, New Scientist 28, 52 (1990).

[21] B. Haisch, A. Rueda, and H. E. Puthoff, Physical Review A 49, 678 (1994).

[22] B. Haisch, A. Rueda, and H. E. Puthoff, Speculations in Science and Technology 20, 99 (1997).

[23] J. F. Woodward and T. Mahood, Foundations of Physics 29, 899 (1999).

[24] J. F. Woodward, Foundations of Physics 31, 819 (2001).

[25] Y. Dobyns, A. Rueda, and B. Haisch, Foundations of Physics 30, 59 (2000).

[26] B. Kosyakov, J. Phys. A 41, 465401 (2008).

[27] K. Lechner, Journal of Mathematical Physics 56, 022901 (2015).

[28] J. Montes, Canadian Journal of Physics 95, 770 (2017). 